\title{
Research on Novel Denoising Method of Variational Mode Decomposition in MEMS Gyroscope
}

\author{
Xiaolei Wang ${ }^{1}$, Huiliang $\mathrm{Cao}^{2}$, Yuzhao Jiao ${ }^{1}$, Taishan Lou ${ }^{1}$, Guoqiang Ding ${ }^{1}$, Hongmei Zhao ${ }^{1}$, Xiaomin Duan ${ }^{3 *}$ \\ 1 College of Electrical and Information Engineering, Zhengzhou University of Light Industry, Dongfeng Road, No.5, \\ Zhengzhou 450002, People's Republic of China,wangxiaolei@zzuli.edu.cn \\ ${ }^{2}$ National Key Laboratory for Electronic Measurement Technology, School of Instrument and Electronics, North University \\ of China, Xueyuan Road, No.3, Taiyuan 030051, People's Republic of China, caohuiliang1986@126.com \\ ${ }^{3}$ School of Electronic Science and Engineering, University of Electronic Science and Technology of China, Chengdu, 611731, \\ People's Republic of China,dxm@uestc.edu.cn
}

\begin{abstract}
The noise signal in the gyroscope is divided into four levels: sampling frequency level, device bandwidth frequency level, resonant frequency level, and carrier frequency level. In this paper, the signal in the dual-mass MEMS gyroscope is analyzed. Based on the variational mode decomposition (VMD) algorithm, a novel dual-mass MEMS gyroscope noise reduction method is proposed. The VMD method with different four-level center frequencies is used to process the original output signal of the MEMS gyroscope, and the results are analyzed by the Allan analysis of variance, which shows that the ARW of the gyroscope is increased from $1.998^{*} 10^{-10} / \sqrt{\mathrm{h}}$ to $1.552^{*} 10^{-40} / \sqrt{\mathrm{h}}$, BS increased from $2.5261^{\circ} / \mathrm{h}$ to $0.0093 \% \mathrm{~h}$.
\end{abstract}

Keywords: MEMS gyroscope, variational mode decomposition, denoising, frequency band.

\section{INTRODUCTION}

The MEMS gyroscope has advantages in small size, light weight, low cost, low power consumption and high reliability, and is suitable for small navigation systems [1]-[9]. With the development of modern science and technology and needs, the accuracy of MEMS gyroscope measurement requirements is getting higher and higher [10], [11]. However, temperature, vibration, space constraints and other factors will affect the gyroscope accuracy, so data processing methods are employed to improve gyroscope accuracy. In work [3], a temperature compensation method for MEMS gyroscope is investigated based on TFPF-MEA-BP (time frequency peak filter, mind evolutionary algorithm, and BP neural network) and the precision of the gyroscope is improved by three orders of magnitude. Reference [12] developed a nonlinear complementary filtering algorithm that provides gyro bias estimation and better estimation results. Both numerical simulation and practical experiments verify the effectiveness of the proposed method. The results show that the denoising performance of SEEMD-TFPF (sample entropy empirical mode decomposition and time-frequency peak filtering) is better than the TFPF method of traditional wavelet Kalman filter and fixed window length in [13]. Work [14] proposed a method to reduce MEMS gyroscope noise by using wavelet filter and CPSO (Chaos Particle Swarm Optimization). A GA (genetic algorithm) based Elman neural network was introduced in work [15] to establish the temperature drift model of the MEMS gyroscope, and the experimental results showed the effectiveness of the proposed GA-Elman based method. Another MEMS gyroscope temperature compensation method based on RBF and NN-GA-KF was proposed in [16], and a three axes MEMS gyroscope achieved a better solution during wide temperature range. A parallel processing algorithm of temperature and noise error for the MEMS gyroscope was proposed in literature [17], and the results showed the noising level was clearly improved. Kalman filter (KF) is a representative algorithm for gyroscope denoising for practical inertial navigation and integrated navigation application [18], however, the filter models and noise characteristics will influence the performance easily [19]. The literature [20] describes the architecture and experimental verification of the automatic pattern matching system using the phase relationship between the residual quadrature and driving the signal in the gyroscope to achieve and maintain the matching resonant mode frequency, the experimental results show that the bias instability of the MEMS under study and the ARW performance gyroscope are improved. Work [21] applied the VMD method to reduce the noise of a MEMS high-G shock accelerometer, and excellent denoising effect was obtained.

DOI: $10.2478 / \mathrm{msr}-2021-0003$ 
Therefore, this paper employs the VMD algorithm, in combination with the different signal frequency characteristics of the MEMS gyroscope to adjust the corresponding bandwidth constraints, study the relationship between the attenuation of the useful signal of each MEMS gyroscope band, then the correctness of the method is verified by the Allan variance.

\section{MEMS GYROSCOPE INTRODUCTION}

\section{A. MEMS gyroscope structure}

The MEMS gyroscope employed in this paper is dual-mass and decoupled style, which utilizes capacitance detection and electrostatic drive method. The mechanical model of MEMS gyroscope structure is shown in Fig.1., and its first four resonant modes with their frequency parameters are shown in Fig.2. The sensing anti-phase mode (3rd mode) and drive anti-phase mode (4th mode) are the working modes of the MEMS gyroscope.

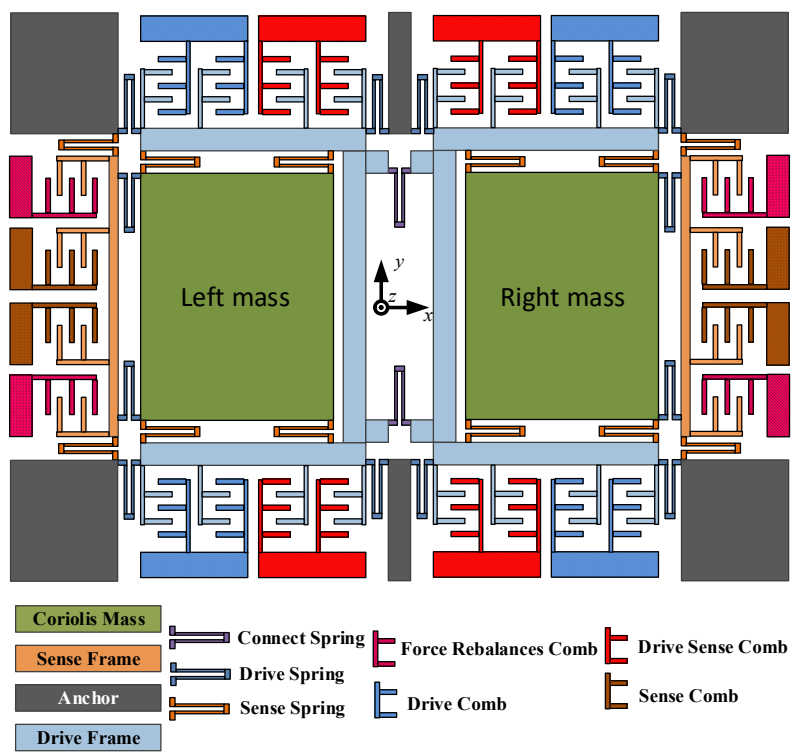

Fig.1. Mechanical model of MEMS gyroscope structure.
The gyroscope structure is formed by left and right parts, the two parts are connected by two connect springs. The left and right Coriolis mass are supported by 2 drive and 4 sense springs, respectively. The whole architecture is suspended by 8 drive and 4 sense springs and the springs are linked to six anchors.

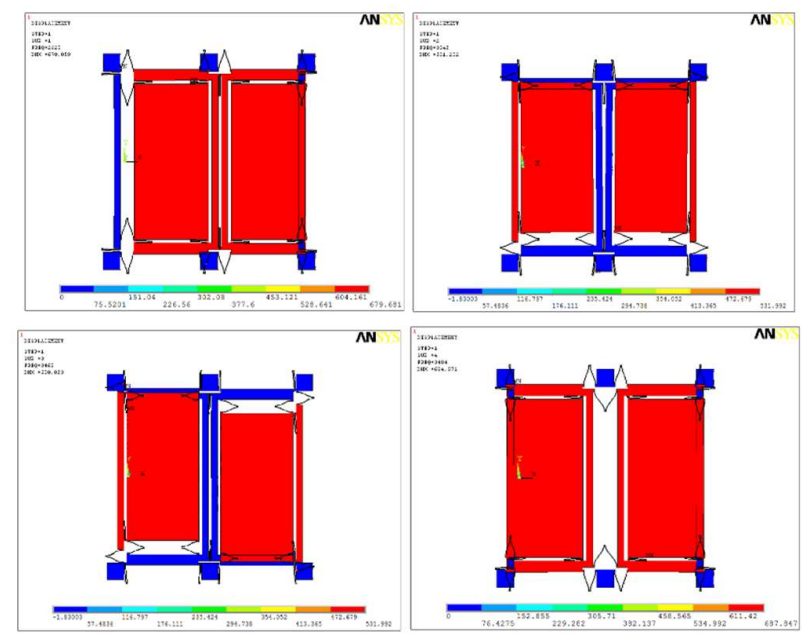

Fig.2. a) Drive in phase mode ( 1 st mode), $\omega_{x l}=2623 \times 2 \pi \mathrm{rad} / \mathrm{s}$; b) Sensing in phase mode ( 2 nd mode), $\omega_{y l}=3342 \times 2 \pi \mathrm{rad} / \mathrm{s}$; c) Sensing anti-phase mode ( $3 \mathrm{rd}$ mode), $\omega_{y 2}=3468 \times 2 \pi \mathrm{rad} / \mathrm{s}$; d) Drive anti-phase mode (4th mode), $\omega_{x 2}=3484 \times 2 \pi \mathrm{rad} / \mathrm{s}$.

\section{B. MEMS gyroscope movement equation}

The structure motion equation can be expressed as in equation (1)[22], where, $m_{x}, m_{y}$, and $m_{c}$ are drive mode mass, sense mode mass, and Coriolis mass; $x, y_{1}$, and $y_{2}$ are drive mode displacement, sense in-phase mode displacement, and anti-phase mode displacement; $Q_{x 2}, Q_{y 1}$, and $Q_{y 2}$ are the quality factors of drive mode, sense in-phase, and anti-phase modes, respectively; $\Omega_{z}$ is angular rate input; $m_{y} \approx m_{c} ; F_{d}$ and $\omega_{d}$ are stimulating magnitude and frequency of drive mode; sense mode displacement $y=y_{1}+y_{2}$, and usually $\omega_{d}=\omega_{x 2}$. And because the Coriolis force is generated by the movement of the drive mode, the force is applied equally both in sense in-phase and sense anti-phase modes. Then, the structure mechanical sensitivity is shown in equation (2), and the frequency difference is a key element:

$$
\begin{gathered}
{\left[\begin{array}{ccc}
m_{x} & 0 & 0 \\
0 & m_{y} & 0 \\
0 & 0 & m_{y}
\end{array}\right]\left[\begin{array}{l}
\ddot{x} \\
\ddot{y}_{1} \\
\ddot{y}_{2}
\end{array}\right]+\left[\begin{array}{ccc}
\frac{\omega_{x 2} m_{x}}{Q_{x 2}} & 0 & 0 \\
0 & \frac{\omega_{y 1} m_{y}}{Q_{y 1}} & 0 \\
0 & 0 & \frac{\omega_{y 2} m_{y}}{Q_{y 2}}
\end{array}\right]\left[\begin{array}{l}
\dot{x} \\
\dot{y}_{1} \\
\dot{y}_{2}
\end{array}\right]+\left[\begin{array}{ccc}
\omega_{x 2}^{2} m_{x} & 0 & 0 \\
0 & \omega_{y 1}^{2} m_{y} & 0 \\
0 & 0 & \omega_{y 2}^{2} m_{y}
\end{array}\right]\left[\begin{array}{l}
x \\
y_{1} \\
y_{2}
\end{array}\right]=} \\
{\left[\begin{array}{l}
F_{d} \sin \left(\omega_{d} t\right) \\
-2 m_{c} \Omega_{z} \dot{x} \\
-2 m_{c} \Omega_{z} \dot{x}
\end{array}\right]}
\end{gathered}
$$




$$
\begin{aligned}
S_{m e} \approx & \frac{-F_{d} Q_{x 2}}{m_{x} \omega_{d}^{2}}\left(\frac{1}{\omega_{y 1}-\omega_{x 2}}+\frac{1}{\omega_{y 2}-\omega_{x 2}}\right) \\
& =-A_{x}\left(\frac{1}{\Delta \omega_{1}}+\frac{1}{\Delta \omega_{2}}\right)
\end{aligned}
$$

\section{MEMS gyroscope monitoring system}

The MEMS gyroscope monitoring system contains three loops: drive loop, sense loop, and feedback loop, which are shown in Fig.3. In drive closed loop, the displacement of drive frame $x(t)$ is detected by drive sensing combs and picked up by a differential amplifier. Then, the phase of the signal is delayed by $90^{\circ}$ to satisfy the phase requirement of AC drive signal $V_{d a c} \operatorname{Sin}\left(\omega_{d} t\right)$. After that, the amplitude of this signal is picked up and is compared with $V_{\text {ref }}$. Next, drive closed loop PI controller generates the control signal, which is modulated by $V_{d a c} \operatorname{Sin}\left(\omega_{d} t\right)$, and then the signal is superposed by $V_{D C}$ to stimulation drive mode.

In sense loop, the left and right Coriolis masses' movement signals are detected and output. Then the signal is enlarged and generates the sense mode movement signal, which is demodulated by signal $V_{d a c} \operatorname{Sin}\left(\omega_{d} t\right)$ and passes through the low pass filter and forms the sense feedback signal. And the controller is employed to calculate the control signal and made as the gyroscope output signal, which is modulated with $V_{d a c} \operatorname{Sin}\left(\omega_{d} t\right)$ to form the AC feedback signal. Finally, DC voltage $V_{F D C}$ is superposed with the AC feedback signal to generate the final feedback signal.

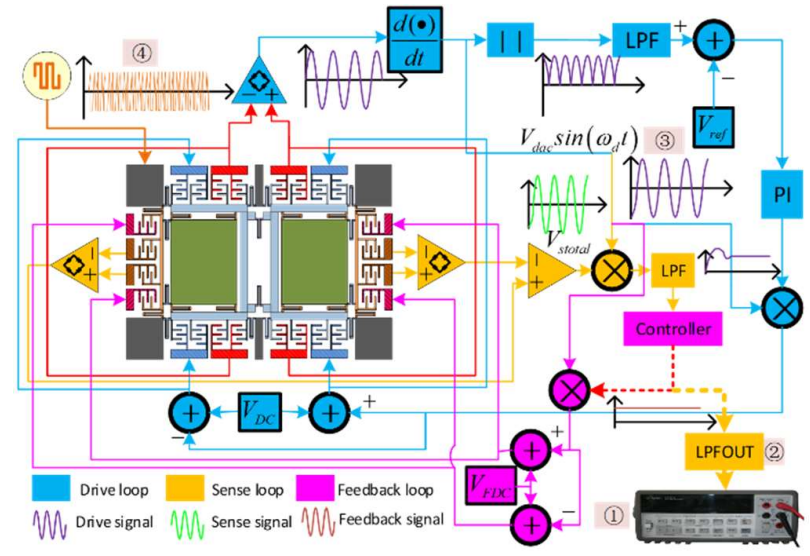

Fig.3. MEMS gyroscope monitoring system.

The MEMS gyroscope structure and prototype photos are shown in Fig.4. The signal in monitoring system can be divided into four levels: the sampling signal (sampling frequency, $1 \mathrm{~Hz}$, (1) in Fig.3.), the bandwidth signal (bandwidth frequency, $100 \mathrm{~Hz}$, (2) in Fig.3.), the resonance signal (resonant frequency, $3.468 \mathrm{kHz}$, (3) in Fig.3.), and the carrier signal (carrier frequency, $10 \mathrm{MHz}$, (4) in Fig.3.) [23], [24].

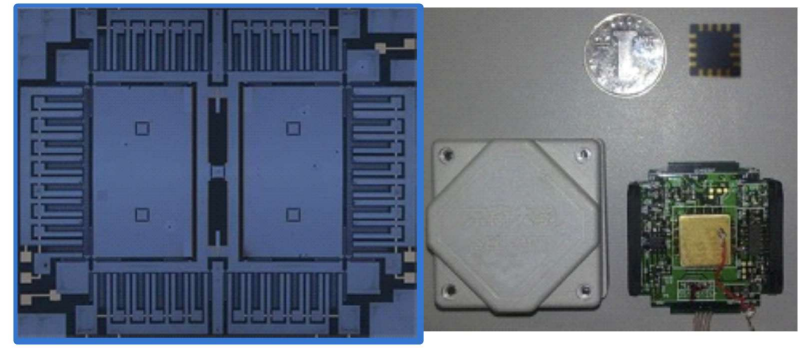

Fig.4. MEMS gyroscope structure and prototype photo.

\section{VMD DENOISING METHOD}

VMD is a new method of adaptive signal processing that decomposes a signal into an intrinsic mode function of different frequencies. The VMD decomposition process is to compute the bandwidth of narrow-band components as follows [25], [26]:

$$
\begin{gathered}
\min _{\left\{u_{k}\right\},\left\{\omega_{k}\right\}}\left\{\sum_{k}\left\|\partial_{t}\left[\left(\delta(t)+\frac{j}{\pi t}\right) * u_{k}(t)\right] e^{-j \omega_{k} t}\right\|_{2}^{2}\right\} \\
\text { s.t. } \sum_{k} u_{k}=f
\end{gathered}
$$

where, $\left\{u_{k}\right\}=\left\{u_{1}, \ldots, u_{K}\right\}$ and $\left\{\omega_{k}\right\}=\left\{\omega_{1}, \ldots, \omega_{K}\right\}$ are the set and center frequencies of all mode components, $\delta(t)$ is Dirac distribution function, $f$ is the original signal. The Lagrangian multiplier $\lambda$ and a quadratic penalty term $\alpha$ can be used to convert (3) into an unconstrained optimization problem as follows:

$$
\begin{aligned}
& L\left(\left\{u_{k}\right\},\left\{\omega_{k}\right\}, \lambda\right) \\
& =\alpha \sum_{k}\left\|\partial_{t}\left[\left(\delta(t)+\frac{j}{\pi t}\right) * u_{k}(t)\right] e^{-j \omega_{k} t}\right\|_{2}^{2} \\
& +\left\|f(t)-\sum_{k} u_{k}(t)\right\|_{2}^{2}+\left\langle\lambda, f(t)-\sum_{k} u_{k}(t)\right\rangle
\end{aligned}
$$

The estimation of narrow-band components in frequencydomain and their center frequencies can be expressed as:

$$
\begin{gathered}
\hat{u}_{k}^{n+1}(\omega)=\frac{\hat{f}(\omega)-\sum_{i \neq k} \boldsymbol{u}_{k}(\omega)+(\hat{\lambda}(\omega) / 2)}{1+2 \alpha\left(\omega-\omega_{k}\right)^{2}} \\
\omega_{k}^{n+1}=\frac{\int_{0}^{\infty} \omega\left|\hat{u}_{k}(\omega)\right|^{2} d \omega}{\int_{0}^{\infty}\left|\hat{u}_{k}(\omega)\right|^{2} d \omega}
\end{gathered}
$$

The VMD algorithm can be summarized in the following steps: 
a) Initialization parameters $\left\{\hat{u}_{k}^{1}\right\},\left\{\hat{w}_{k}^{1}\right\}, \hat{\lambda}^{1}, n \leftarrow 0$;

b) $n \leftarrow n+1$, Run the entire cycle;

c) Execute the inner loop $k \leftarrow k+1$ until $k=K$, update $u_{k}(t)$ : $u_{k}^{n+1} \leftarrow \arg \operatorname{minL}\left(\left\{u_{i<k}^{n+1}\right\},\left\{u_{i \geq k}^{n}\right\},\left\{\omega_{i}^{n}\right\}, \lambda^{n}\right)$;

d) Execute the inner loop $k \leftarrow k+1$ until $k=K$, update $w_{k}(t)$ : $w_{k}^{n+1} \leftarrow \underset{w_{k}}{\arg \operatorname{minL}}\left(\left\{u_{i}^{n+1}\right\},\left\{w_{i<k}^{n+1}\right\},\left\{w_{i \geq k}^{n}\right\}, \lambda^{n}\right) ;$

e) Perform a dual ascent for all $\omega \geq 0$, and obtain $\hat{\lambda}^{n+1}(\omega)$ as: $\hat{\lambda}^{n+1}(\omega) \leftarrow \hat{\lambda}^{n}(\omega)+\tau\left(\hat{f}(\omega)-\sum_{k} \hat{u}_{k}^{n+1}(\omega)\right)$;

f) Repeat step $\boldsymbol{b})$-e $)$ and until $\sum_{k}\left(\left\|\hat{u}_{k}^{n+1}-\hat{u}_{k}^{n}\right\|_{2}^{2} /\left\|\hat{u}_{k}^{n}\right\|_{2}^{2}\right) \prec \varepsilon$ is satisfied;

g) Stop all loops and output the result, and then get K narrowband IMF component.

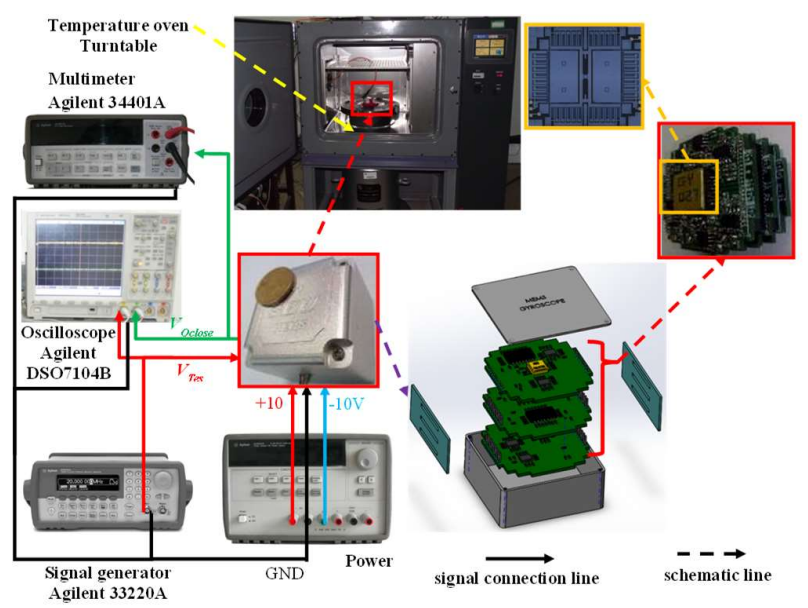

Fig.5. Experiment platform.

\section{EXPERIMENT AND RESULTS}

\section{A. Experiment plant form}

The experiment plant form includes temperature oven turntable which provides the MEMS gyroscope with stable temperature and the static movement $\left(\Omega_{z}=0\right)$ testing environment. The power supply is $\pm 10 \mathrm{~V}$ (with digital power Agilent E3631A), and the output signal is picked up by both multimeter (with Agilent 34401A) and oscilloscope (with Agilent DSO7104B). The signal generator is employed to debug the monitoring system. The experiment plant form is shown in Fig.5.

The original data and the denoising results are shown in Fig.6., where black refers to the original output signal, blue refers to the output signal of the sampling bandwidth limit, green refers to the output signal of the bandwidth signal limitation, yellow refers to the output signal of the resonance bandwidth limitation, and red refers to the output signal of the carrier bandwidth limit. It is obvious that the carrier frequency level denoising method achieves better results.
Then the Allan variance analysis of denoising results is shown in Fig.7., the bias instability (BS) and angular random walk (ARW) of four frequency levels are shown in Table 1.
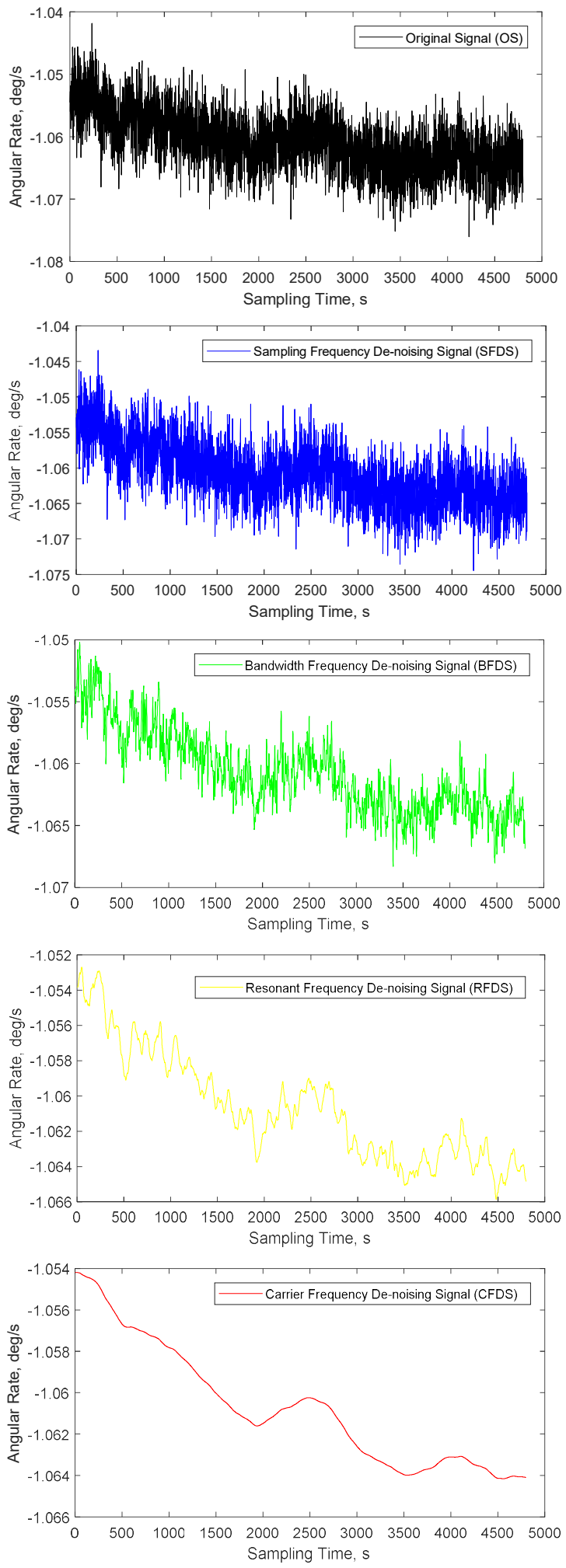

Fig.6. Comparisons of denoising results. 
Table 1. Allan variance analysis of denoising results.

\begin{tabular}{|c||c|c||c|c|}
\hline DATA & $\begin{array}{c}\text { Bias Stability } \\
{[\% / \mathbf{h}]}\end{array}$ & $\begin{array}{c}\text { Improve } \\
{[\%]}\end{array}$ & $\begin{array}{c}\text { Angular Radom } \\
\text { Walking }[\% \sqrt{\mathbf{h}]}\end{array}$ & $\begin{array}{c}\text { Improve } \\
{[\%]}\end{array}$ \\
\hline \hline OS & 2.5261 & - & $1.998 * 10^{-1}$ & - \\
\hline SFDS & 2.3632 & 6.45 & $1.581 * 10^{-1}$ & 20.87 \\
\hline BFDS & 0.9321 & 63.10 & $1.554 * 10^{-2}$ & 92.22 \\
\hline RFDS & 0.0866 & 96.57 & $1.443 * 10^{-3}$ & 99.28 \\
\hline CFDS & 0.0093 & 99.63 & $1.552 * 10^{-4}$ & 99.92 \\
\hline
\end{tabular}

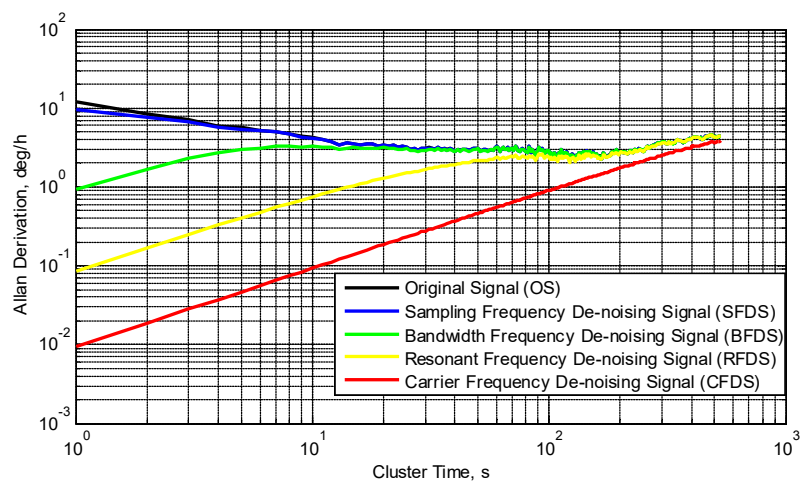

Fig.7. Allan variance analysis of denoising results.

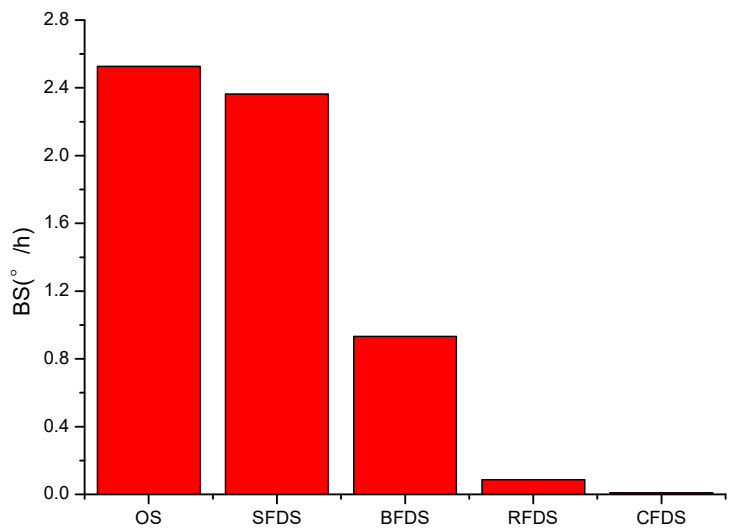

Fig.8. Bias Stability of denoising results.

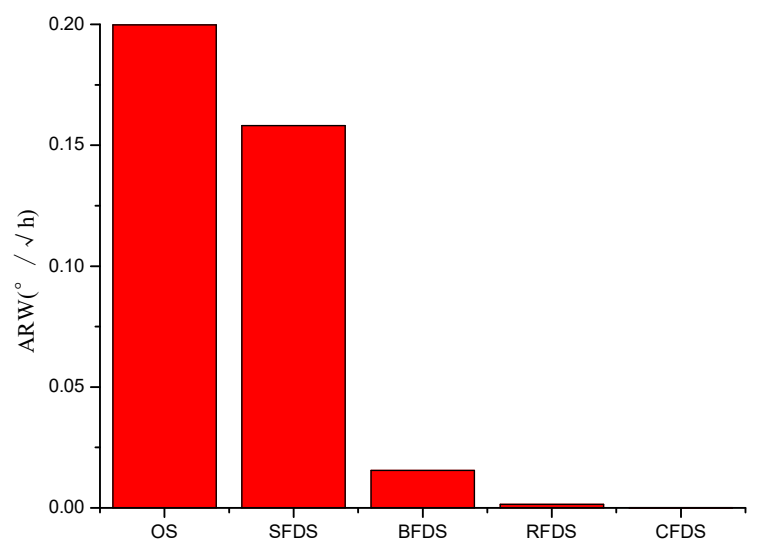

Fig.9. Angular Radom Walking of denoising results.

\section{CONCLUSION}

In this study, the VMD algorithm was used in combination with the bandwidth characteristics of the MEMS gyroscope to adjust the corresponding bandwidth limitation. The relationship between the attenuation of each useful signal of the MEMS gyroscope is studied and verified by the Allan variance. The experimental results show that the MEMS gyroscope has the highest attenuation of the output signal and the carrier signal, and the ARW of the gyroscope improves from $1.998^{*} 10^{-10} / \sqrt{ } \mathrm{h}$ to $1.552 * 10^{-40} / \sqrt{ } \mathrm{h}$, the $\mathrm{BS}$ improves from $2.5261^{\circ} / \mathrm{h}$ to $0.0093^{\circ} / \mathrm{h}$, respectively. This method is helpful to the practical application of MEMS inertial sensors.

\section{ACKNOWLEDGMENT}

This work is supported by National Natural Science Foundation of China (No. 51705477, 51635011, 51727808, 51922009), and Pre-Research Field Foundation of Equipment Development Department of China No. 61405170104. The research is also supported by the program for the Top Young Academic Leaders of Higher Learning Institutions of Shanxi, Fund Program for the Scientific Activities of Selected Returned Overseas Professionals in Shanxi Province, Shanxi Province Science Foundation for Youths (No. 201801D221195), Young Academic Leaders of North University of China (No. QX201809), Shanxi Province patent promotion and implementation program of Shanxi Province (2019025), the Weapons and Equipment Joint Fund (No. 6141B021304), and the Fund for Shanxi "1331 Project" Key Subjects Construction.

\section{REFERENCES}

[1] Shen, C., Liu, X., Cao, H., Zhou, Y., Liu, J., Tang, J., Guo, X., Huang, H., Chen, X. (2019). Brain-like navigation scheme based on MEMS-INS and place recognition. Applied Sciences, 9 (8), 1708.

[2] Zhang, Y., Liu, W., Yang, X., Xing, S. (2015). Hidden Markov model-based pedestrian navigation system using MEMS inertial sensors. Measurement Science Review, 15 (1), 35-43.

[3] Cao, H., Cui, R., Liu, W., et al. (2021). Dual mass MEMS gyroscope temperature drift compensation based on TFPF-MEA-BP algorithm. Sensor Review, DOI: 10.1108/SR-09-2020-0205. (in press)

[4] Guo, X., Tang, J., Li, J., Shen, C., Liu, J. (2019). Attitude measurement based on imaging ray tracking model and orthographic projection with iteration algorithm. ISA Transactions, 95, 379-391.

[5] Mellal, I., Laghrouche, M., Bui, H. (2017). Field programmable gate array (FPGA) respiratory monitoring system using a flow microsensor and an accelerometer. Measurement Science Review, 17 (2), 61-67.

[6] Shen, C., Zhang, Y., Tang, J., Cao, H., Liu, J. (2019). Dual-optimization for a MEMS-INS/GPS system during GPS outages based on the cubature Kalman filter and neural networks. Mechanical Systems and Signal Processing, 133, 106222. 
[7] Dichev, D., Koev, H., Bakalova, T., Louda, P. (2014). A gyro-free system for measuring the parameters of moving objects. Measurement Science Review, 14 (5), 263-269.

[8] Cui, J., Zhao, Q., Yan, G. (2019). Effective bias warmup time reduction for MEMS gyroscopes based on active suppression of the coupling stiffness. Microsystems \& Nanoengineering, 5 (1).

[9] Zhou, W., Chen, L., Yu, H., Chen, Y. (2016). Sensitivity jump of micro accelerometer induced by micro-fabrication defects of micro folded beams. Measurement Science Review, 16 (4), 228-234.

[10] Cao, H., Li, H., Shao, X., Liu, Z., Kou, Z., Shan, Y., Shi, Y., Shen, C., Liu, J. (2018). Sensing mode coupling analysis for dual-mass MEMS gyroscope and bandwidth expansion within wide-temperature range. Mechanical Systems \& Signal Processing, 98, 448-464.

[11] Cao, H., Liu, Y., Kou, Z., Zhang, Y., Shao, X., Gao, J., Huang, K., Shi, Y., Tang, J., Shen, C., Liu, J. (2019). Design, fabrication and experiment of double U-beam MEMS vibration ring gyroscope. Micromachines (Basel), 10 (3), 186.

[12] Wu, Z., Sun, Z., Zhang, W., Chen, Q. (2016). A novel approach for attitude estimation based on MEMS inertial sensors using nonlinear complementary filters. IEEE Sensors Journal, 16 (10), 3856-3864.

[13] Shen, C., Li, J., Zhang, X., Shi, Y., Tang, J., Cao, H., Liu, J. (2016). A noise reduction method for dual-mass micro-electromechanical gyroscopes based on sample entropy empirical mode decomposition and timefrequency peak filtering. Sensors, 16 (6), 796.

[14] Xing, H., Hou, B., Lin, Z., Guo, M. (2017). Modeling and compensation of random drift of MEMS gyroscopes based on least squares support vector machine optimized by chaotic particle swarm optimization. Sensors, 17 (10), 2335.

[15] Shen, C., Song, R., Li, J., Zhang, X., Tang, J., Shi, Y., Liu, J., Cao, H. (2016). Temperature drift modeling of MEMS gyroscope based on genetic-Elman neural network. Mechanical Systems and Signal Processing, 72-73, 897-905.

[16] Cao, H., Zhang, Y., Shen, C., Liu, Y., Wang, X. (2018). Temperature energy influence compensation for MEMS vibration gyroscope based on RBF NN-GA-KF method. Shock and Vibration, 2018, 2830686.
[17] Shen, C., Yang, J., Tang, J., Liu, J., Cao, H. (2018). Note: Parallel processing algorithm of temperature and noise error for micro-electro-mechanical system gyroscope based on variational mode decomposition and augmented nonlinear differentiator. Review of Scientific Instruments, 89, 076107.

[18] Xu, Q., Li, X., Chan, C.Y. (2017). A cost-effective vehicle localization solution using an interacting multiple model-unscented Kalman filters (IMM-UKF) algorithm and grey neural network. Sensors, 17, 1431.

[19] Kownacki, C. (2011). Optimization approach to adapt Kalman filters for the real-time application of accelerometer and gyroscope signals' filtering. Digital Signal Processing, 21, 131-140.

[20] Sonmezoglu, S., Alper, S.E., Akin, T. (2014). An automatically mode-matched MEMS gyroscope with wide and tunable bandwidth. Journal of Microelectromechanical Systems, 23 (2), 284-297.

[21] Cao, H., Zhang, Z., Zheng, Y., Guo, H., Zhao, R., Shi, Y., Chou, X. (2021). A new joint denoising algorithm for High-G calibration of MEMS accelerometer based on VMD-PE-wavelet threshold. Shock and Vibration, 2021, 8855878.

[22] Cao, H., Zhang, Y., Han, Z., Shao, X., Gao, J., Huang, K., Shi, Y., Tang, J., Shen, C., Liu, J. (2019). Pole-zero temperature compensation method for sensing mode coupling dual-mass MEMS gyroscope bandwidth expansion. IEEE-ASME Transactions on Mechatronics, 24 (2), 677-688.

[23] Cao, H., Li, H., Liu, J., Shi, Y., Tang, J., Shen, C. (2016). An improved interface and noise analysis of a turning fork microgyroscope structure. Mechanical Systems and Signal Processing, 70-71, 1209-1220.

[24] Cao, H., Liu, Y., Zhang, Y., Shao, X., Gao. J., Huang, K., Shi, Y., Tang, J., Shen, C., Liu, J. (2019). Design and experiment of dual-mass MEMS gyroscope sense closed system based on bipole compensation method. IEEE Access, 7, 49111-49124.

[25] Dragomiretskiy, K., Zosso, D. (2014). Variational mode decomposition. IEEE Transactions on Signal Processing, 62 (3), 531-544.

[26] Upadhyay, A., Pachori, R.B. (2017). Speech enhancement based on mEMD-VMD method. Electronics Letters, 53 (7), 502-504. 